OPEN ACCESS

Edited by:

Felice lasevoli,

University of Naples Federico II, Italy

Reviewed by:

Heather Van Mater,

Duke University, United States

NIcola Warren

Metro South Addiction and Mental

Health Services, Australia

Bastien Joubert,

Hospices Civils de Lyon, France

*Correspondence:

Jinfang $\mathrm{Li}$

361385724@qq.com

Tianfeng Zhang

ztf075502@163.com

tThese authors have contributed equally to this work

Specialty section:

This article was submitted to

Schizophrenia,

a section of the journal

Frontiers in Psychiatry

Received: 08 September 2021

Accepted: 20 January 2022

Published: 23 February 2022

Citation:

Wang J, Gong S, Kong F, Cai D,

Huang $B$, Zheng $H$, Lin S, Li J and Zhang $T$ (2022) Psychotic Symptoms

as the Initial Presentation of a

Long-Lasting Misdiagnosed

Anti-GAD65 Autoimmune

Encephalitis: An Emblematic Case and

Literature Review.

Front. Psychiatry 13:754938.

doi: 10.3389/fpsyt.2022.754938

\section{Psychotic Symptoms as the Initial Presentation of a Long-Lasting Misdiagnosed Anti-GAD65 Autoimmune Encephalitis: An Emblematic Case and Literature Review}

\author{
Jianjun Wang ${ }^{1,2,3 t}$, Shenglan Gong ${ }^{1,4,5 t}$, Fanxin Kong ${ }^{1,2 \dagger}$, Dongbin Cai ${ }^{1,2}$, Binqing Huang ${ }^{1,2}$, \\ Haotao Zheng ${ }^{1,2}$, Songjun Lin ${ }^{1,2}$, Jinfang $\mathrm{Li}^{1,2 *}$ and Tianfeng Zhang ${ }^{4,5 *}$ \\ ${ }^{1}$ Fourth Clinical Medical College, Guangzhou University of Chinese Medicine, Shenzhen, China, ${ }^{2}$ Department of Neurology \\ and Psychology, Shenzhen Traditional Chinese Medicine Hospital, Shenzhen, China, ${ }^{3}$ Global Clinical Scholars Research \\ Training (GCSRT), Harvard Medical School, Boston, MA, United States, ${ }^{4}$ Shenzhen Hospital (Futian) of Guangzhou University \\ of Chinese Medicine, Shenzhen, China, ${ }^{5}$ Sixth Clinical Medical College, Guangzhou University of Chinese Medicine, \\ Shenzhen, China
}

Objective: To present a long-lasting misdiagnosed case of anti-GAD65 autoimmune encephalitis (AE) and promote the early identification of reversible psychotic symptoms in $\mathrm{AE}$.

Methods: The case report was generated through detailed assessment of clinical characteristics, cerebral magnetic resonance images, and laboratory results. Meanwhile, a literatures review related to the topic was conducted.

Results: Psychotic symptoms could be presented in the early stage of anti-GAD65 autoimmune encephalitis. Even though there exists a transdisciplinary gap that hinder the timely recognition of early psychiatric symptoms as components of organic disease, a few strategies could be introduced to enable the earlier recognition and appropriate treatment.

Conclusions: Our report intends to raise awareness to promote the early identification of immune-mediated "symptomatic" forms of psychosis.

Keywords: autoimmune, encephalitis, psychosis, GAD65, autoimmune hepatitis, autoantibodies, seizure (medical records)

\section{INTRODUCTION}

Autoimmune encephalitis (AE) is a rare, debilitating and potentially fatal immune-mediated disease characterized by seizures, cognitive impairment, and psychiatric symptoms $(1,2)$. The psychiatric symptoms were prominent when AE was recognized more than a decade ago (3). ManeDamas et al. claimed that patients with AE showed marked psychotic symptoms prior to overt neurological manifestations and were admitted to a psychiatric hospital $(4,5)$. In addition, the psychotic manifestations are similar to those observed in primary functional psychiatric disorders, making misdiagnosis or underdiagnosis occur in AE patients with psychotic symptoms. 
Glutamic acid decarboxylase 65 (GAD65) antibody is a subtype of new intracellular antigens and associated with limbic encephalitis (LE) $(6,7)$. As core manifestations of LE, cognition impairments and seizures have been well-described in patients with GAD65 encephalitis (6-8), whereas psychiatric symptoms remain an open topic. Since GAD was hypothesized as the ratelimiting enzyme in the synthesis of $\gamma$-aminobutyric acid (GABA), and dysfunctions of GABAergic neurons were associated with the pathogenesis of psychiatric manifestations $(6,9,10)$, it is worthwhile to explore the psychiatric manifestations that would complicate the diagnosis of GAD65 encephalitis.

In this case report, we reported a Chinese female who initially showed episodic psychotic symptoms and was finally diagnosed as GAD65 encephalitis. The comprehensive neurological and psychiatric characteristics were assessed and the potential clues for early diagnosis were emphasized. The informed consent was obtained from the patient. Furthermore, a literature review related to the topic was conducted.

\section{CASE DESCRIPTION}

Mrs. $\mathrm{K}$ is a 30-year-old female, who initially suffered from insomnia, auditory hallucinations, and behavioral abnormalities (e.g., grabbing food with hands) 2 years ago. These symptoms were initially sporadic and gradually progressed with episodes of generalized tonic-clonic seizures, which lasted for 3 to $5 \mathrm{~min}$ and occurred 1 to 2 times a month. According to her chart, two past admissions for similar recurrent seizures and behavior deficits were recorded in two general hospitals. The electroencephalogram (EEG) results demonstrated that the rhythmic epileptiform discharges arose out of the bilateral temporal regions with right predominance. The thyroid hormones, folate, B12 vitamin and serum sodium concentrations were normal. The routine cerebrospinal fluid (CSF) analysis (including oligoclonal bands) and brain magnetic resonance imaging (MRI) scan without contrast were unremarkable. Therefore, she was diagnosed with epilepsy (ICD-10 Code, G 40) and suspicious encephalitis (ICD-10 Code, G 04.9) in the two previous hospitals, respectively. Sodium valproate $(1,000$ $\mathrm{mg} / \mathrm{d})$, carbamazepine $(200 \mathrm{mg} / \mathrm{d})$, and olanzapine $(2.5 \mathrm{mg} / \mathrm{d})$ were prescribed and taken. However, the seizures were only partially alleviated, and obvious behavior abnormalities were progressed in social settings. Moreover, her husband reported that she had paranoid insecurity (e.g., crying or not sleeping) and a quality of imperative auditory hallucination (e.g., kneeling on the road for ancestor worship). Then, she was admitted to a psychiatric hospital, where her symptoms were not successfully controlled with sodium valproate $(1,500 \mathrm{mg} / \mathrm{d})$, carbamazepine $(600 \mathrm{mg} / \mathrm{d})$, topiramate $(200 \mathrm{mg} / \mathrm{d})$, and risperidone $(2 \mathrm{mg} / \mathrm{d})$. Although the frequency of chaotic behaviors and seizures were decreased, she became confused, minimally responsive to directions and communications, and even could not recognize her family members.

The patient was then admitted to our facility in September 2019. The general physical and neurological examinations were unremarkable. The mental status examination revealed
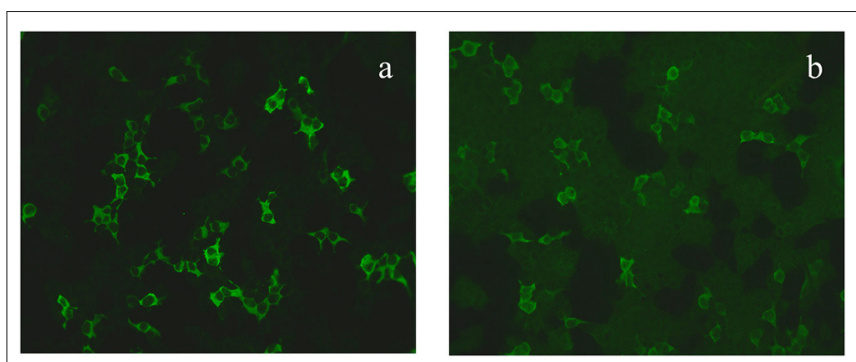

FIGURE 1 | The GAD65 antibody was diluted 1:10 in the serum (a) and 1:100 in the cerebrospinal fluid (b).

blunted effect, slow response, inappropriate laughing or crying, and episodes of auditory hallucination from her dead mother (e.g., telling her to dash into traffic, or that her son got lost). She could not realize these disorganized behaviors until she got relieved by the following treatments. Consistent to previous EEG findings, rhythmic epileptiform discharges were confirmed. Further, a thorough evaluation for organic causes was performed, including brain MRI with contrast, lumbar puncture (LP), an autoimmune workup and positron emission tomography-computed tomography (PET/CT). The serum and CSF antibodies to eight neuronal proteins were assessed, including NMDA-R, GAD65, a-amino-3-hydroxy-5-methyl-4isoxazole prophetic acid receptor $1 / 2$ (AMPAR1/2), gammaaminobutyric acid B receptor (GABA B), leucine-rich glioma inactivated 1 (LGI1) and contacting-associated protein-like 2 (CASPR2), dipeptidyl peptidase protein-like 6 (DPPX), and immunoglobulin-like cell adhesion molecule 5 (IgLON5). The anti-GAD65 antibody titer was positive in CSF $(++1: 10)$ and in serum $(++1: 100)$ (Figures 1a,b). Blood and CSF tests for viral or bacterial infections were negative. It was interesting that a rapid improvement of verbal response and psychiatric symptoms were repeatedly observed after LP. Due to the association of anti-GAD65 autoantibodies with tumors, a whole-body $18 \mathrm{~F}$ fludeoxyglucose PET/CT was further performed, revealing no signs of tumor. The paraneoplastic antibody detection in CSF was also unremarkable. Conventional structural MRI was negative. However, ${ }^{1} \mathrm{H}$-magnetic resonance spectroscopy (MRS) revealed a markedly elevated Cho peak, thus leading to an increased choline (Cho)-to-creatine ( $\mathrm{Cr}$ ) ratio (Cho/Cr: 1.67) in the right hippocampus, compared with the left mirrored hippocampus (Figure 2).

Based on the clinical presentation, EEG and laboratory findings, a GAD65-mediatd autoimmune encephalitis (ICD10 Code, G 04.9) was confirmed. The patient was treated first with a 5-day course of intravenous immunoglobulin $(0.4 \mathrm{~g} / \mathrm{kg})$ and 3 -day course of methylprednisolone $(1,000$ $\mathrm{mg} /$ day), followed by an oral prednisolone therapy (started at a dose of $50 \mathrm{mg}$ /day). The immunosuppressive treatment resulted in a moderate improvement of behavior disturbance and seizures until symptoms deteriorated after months. The patient was then treated with another two cycles of intravenous immunoglobulin and methylprednisolone in the following 

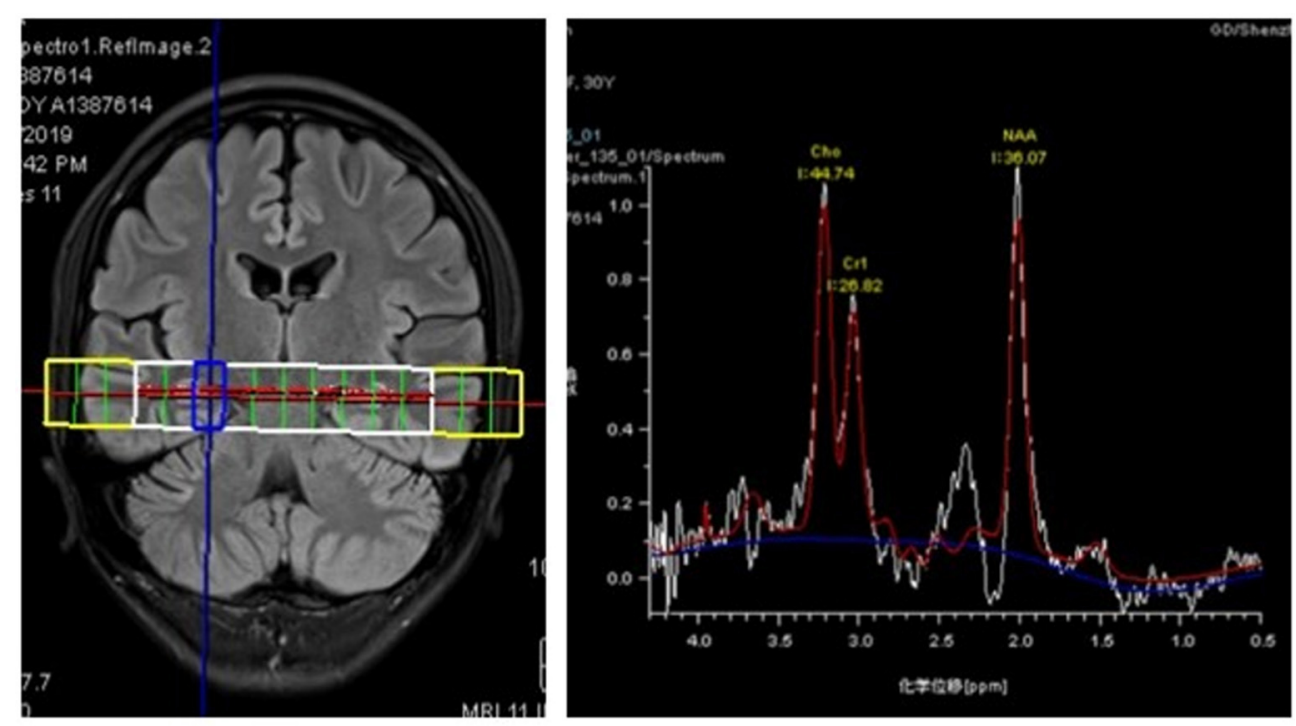

FIGURE $2 \mid{ }^{1} \mathrm{H}$-magnetic resonance spectroscopy (MRS) images showing an increased Cho/Cr ratio (1.67) in the right hippocampus with a markedly elevated Cho peak.

sessions. Moreover, a combination of methylprednisolone (1,000 $\mathrm{mg}$ /day for 3 consecutive days) and monthly intravenous pulses of cyclophosphamide (500 $\mathrm{mg} / \mathrm{m}^{2}$ body surface) was introduced in the latest session. We observed moderate remission after half a year follow-up. She was free of seizures, positive psychotic symptoms, or disorganized behavior with the augmented oral anticonvulsant and antipsychotics (topiramate $125 \mathrm{mg} / \mathrm{d}$, sodium valproate $1,500 \mathrm{mg} / \mathrm{d}$, and olanzapine $2.5 \mathrm{mg} / \mathrm{d}$ ). Although she experienced frequent insomnia, slow response, and blunted effect, preventing her from working properly, she could stay at home alone and take care of herself. The score on the modified Rankin scale was reduced from 4 to 2 .

\section{DISCUSSION}

Although this patient presented typical features of LE (11), unremarkable findings from routine MRI scan, behavior disturbance, and acute psychotic episodes contributed to the delayed diagnosis. The patient presented psychiatric symptoms as the initial disturbance and responded poorly to antipsychotics, which were not well-recognized in two recent observational studies on $\mathrm{GAD} 5^{+}$encephalitis $(7,8)$. Our case could be served as supplemental evidence to strengthen the early recognition of anti-GAD65 Abs-mediated AE, and to avoid the long-lasting misdiagnosis and delayed immunosuppressive treatment.

LE is usually characterized by short-term memory deficits, seizures, and psychiatric symptoms suggestive of limbic involvement (11). GAD65 Abs has drawn increasing attention as a subtype of intracellular antigens targeting at the limbic system. There have been case series reporting psychotic symptoms related to GAD65 encephalitis $(12,13)$. However, the evidence about the clinical entity could be controversial. A recent meta-analysis reported a pooled prevalence rate of 5.8\% for GAD65 Abs in patients with psychosis (14), which suggested that the psychiatric symptoms are important phenotypes associated with GAD65 Abs and are worthy of attention. In contrast, the psychiatric symptoms were not well-recognized in two recent observational studies on GAD65 ${ }^{+}$encephalitis $(7,8)$, one of which reported that $14.3 \%$ participants $(n=5)$ had behavioral disturbances and stated the deficits of non-standard neuropsychological assessments. Hence, there are increasing debates regarding the comprehensive neuropsychiatric evaluations of psychiatric symptoms in AE (15). On one hand, patients are sometimes first hospitalized in a psychiatric institution as $\mathrm{AE}$ can resemble acute psychosis (2), causing concern about the misdiagnosis of $\mathrm{AE}$ patients with acute psychosis (16). On the other hand, as advocated, psychiatrists should be involved in the diagnosis and treatment of AE patients, since the scarcity of psychiatric investigations could lead to underdiagnosis of psychiatric phenotypes (15). Therefore, regarding the interpretations of the currently available data on the neuropsychiatric features of AE, including anti-GAD65 patients, we should keep in mind the potential misdiagnosis or underdiagnosis in many instances $(2,6)$.

Due to the transdisciplinary gap between the neurology and psychiatry, it could be challenging at times to recognize the early psychiatric symptoms as part of the organic disease. It has been reported that the mean time between the occurrence of first symptoms and antibody testing was often alarmingly prolonged from 74 days to 483 days (17). The duration in our case was delayed for a period of 2 years. Thus, there is a great need to raise awareness of early diagnosis of $\mathrm{AE}$ for both neurologist and psychiatrist. A few strategies could be introduced. On one hand, the hallucinations in this patient were not systematic but with episodic characteristics, which is clearly different from the 
persistent psychotic symptoms in primary psychotic disorder as schizophrenia. On the other hand, the patient had multiple red flags for the suspicion of autoimmune encephalitis $(17,18)$, including seizures, cognitive impairments, insufficient response to antipsychotics, and rapid progression. Additionally, early diagnosis is also urged due to bad outcomes of the delayed immunosuppression treatment. It has been reported that delays in immune therapy administration could lead to treatment resistance and permanent sequelae (19). In our case, the patient received most types of immunotherapies used for $\mathrm{AE}$, including steroids, IVIg, and cyclophosphamide. The treatment seemingly only had moderate effects on the patient's clinical condition. It has been proposed that acute and reversible immune activation in the initial stage of disease could result in significantly better outcomes even without any permanent brain damage $(20,21)$. Thus, early recognition is mandatory for both $\mathrm{AE}$ and newly proposed autoimmune psychosis (18).

Owing to the newly-recognized autoimmune encephalitis mediated by GAD65 Abs, a few interesting phenomena are deserved to be focused on. Firstly, an unexplained clinical improvement after LP was repeatedly observed. Considering the T-cell-based cytotoxicity and the questionable pathogenic significance with intracellular antigens (e.g., GAD65) (22), removing antibody-positive CSF will not have any effects on the antibodies presented in the brain parenchyma. A recent study also reported limited therapeutic effects of immunoadsorption therapy in patients with GAD65 encephalitis (23). It might be infeasible to monitor the antibody titers, even though a decrease of anti-GAD65 concentration was occasionally reported accompanying the clinical improvement (7). Besides, the patient could hardly benefit from the reduced CSF pressure after LP due to the absence of increased CSF pressure or hydrocephalus. Thus, other factors should be fully considered and inspected, such as the frequent presence of coexisting autoantibodies $(13,24)$. Secondly, an increased Cho peak in the right hippocampus was observed in MRS, which suggests cell destruction or high cellularity (25). Consistently, the corresponding rhythmic epileptiform discharges in right hippocampus and the clinical presentation targeting at the limbic area were also observed. Hence, the MRS could be served as an alternative method to observe microstructure alterations in the absence of routine structure MR findings. Meanwhile, considering the characteristics of MRS in glioblastoma (GBM), it could be further used to differentiate GBM from $\mathrm{AE}$ with visible neuroimaging findings in conventional MR scan (26). At last, with the increasing applications of multimodal neuroimaging (e.g., diffusion tensor

\section{REFERENCES}

1. Broadley J, Seneviratne U, Beech P, Buzzard K, Butzkueven H, O'Brien $\mathrm{T}$, et al. Prognosticating autoimmune encephalitis: a systematic review. J Autoimmun. (2019) 96:24-34. doi: 10.1016/j.jaut.2018. 10.014

2. Endres D, Leypoldt F, Bechter K, Hasan A, Steiner J, Domschke K, et al. Autoimmune encephalitis as a differential diagnosis of schizophreniform psychosis: clinical symptomatology, pathophysiology, diagnostic approach, imaging etc.) in autoimmune encephalitis $(27,28)$, a better understanding of the specific pathophysiologic properties of encephalitis is warranted, which would be beneficial to promote the early diagnosis of AE.

Taken together, psychotic symptoms could be presented as a component of GAD65-mediated autoimmune encephalitis, and may lead to misdiagnosis and delayed immunosuppressive treatment. Given the interdisciplinary gap in the literature, it is necessary for neurologists and psychiatrists to be aware of the early identification of immune-mediated "symptomatic" forms of psychosis.

\section{DATA AVAILABILITY STATEMENT}

The original contributions presented in the study are included in the article, further inquiries can be directed to the corresponding authors.

\section{ETHICS STATEMENT}

The studies involving human participants were reviewed and approved by Ethical Committee of Shenzhen Traditional Chinese Medicine Hospital. The patients/participants provided their written informed consent to participate in this study. Written informed consent was obtained from the individual(s) for the publication of any potentially identifiable images or data included in this article.

\section{AUTHOR CONTRIBUTIONS}

JW, SG, JL, and FK observed the patient and analyzed the data. JW, SG, DC, and HZ reviewed the literature. BH, SL, FK, JL, and TZ formulated the conception of the study. JW, SG, and FK wrote the paper. All authors approved the final work.

\section{FUNDING}

This work was supported by National Natural Science Foundation of China (82004284), Guangdong Medical Science Foundation (A2020370 and A2021199), Guangdong Administration of Traditional Chinese Medicine Project (20201419), Shenzhen Science and Technology Research Program (RCBS20200714114959156), and Shenzhen Municipal Health and Family Planning System Scientific Research Project (SZFZ2018013). 270:803-18. doi: 10.1007/s00406-020-01113-2

3. Dalmau J, Tüzün E. Wu Hy, Masjuan J, Rossi JE, Voloschin A, et al. Paraneoplastic anti-N-methyl-D-aspartate receptor encephalitis associated with ovarian teratoma. Annals Neurol. (2007) 61:25-36. doi: 10.1002/ana.21050

4. Kayser MS, Titulaer MJ, Gresa-Arribas N, Dalmau J. Frequency and characteristics of isolated psychiatric episodes in anti-N-methyl-d-aspartate receptor encephalitis. JAMA 
Neurol. (2013) 70:1133-9. doi: 10.1001/jamaneurol.2013. 3216

5. Mane-Damas M, Hoffmann C, Zong S, Tan A, Molenaar PC, Losen $\mathrm{M}$, et al. Autoimmunity in psychotic disorders. Where we stand, challenges and opportunities. Autoimmunity Rev. (2019) 18:102348. doi: 10.1016/j.autrev.2019.102348

6. Gagnon MM, Savard M. Limbic encephalitis associated with GAD65 antibodies: brief review of the relevant literature. Can J Neurol Sci. (2016) 43:486-93. doi: 10.1017/cjn.2016.13

7. Muñoz-Lopetegi A, de Bruijn MA, Boukhrissi S, Bastiaansen AE, Nagtzaam MM, Hulsenboom ES, et al. Neurologic syndromes related to anti-GAD65: clinical and serologic response to treatment. Neurol-Neuroimmunol Neuroinfl. (2020) 7:e696. doi: 10.1212/NXI.0000000000000696

8. Joubert B, Belbezier A, Haesebaert J, Rheims S, Ducray F, Picard G, et al. Long-term outcomes in temporal lobe epilepsy with glutamate decarboxylase antibodies. J Neurol. (2020) 267:2083-9. doi: 10.1007/s00415-020-09807-2

9. Sloviter RS, Dichter MA, Rachinsky TL, Dean E, Goodman JH, Sollas $\mathrm{AL}$, et al. Basal expression and induction of glutamate decarboxylase GABA in excitatory granule cells of the rat and monkey hippocampal dentate gyrus. J Comp Neurol. (1996) 373:593-618. doi: 10.1002/(SICI)10969861(19960930)373:4<593::AID-CNE8>3.0.CO;2-X

10. Mitoma H, Song S-Y, Ishida K, Yamakuni T, Kobayashi T, Mizusawa H. Presynaptic impairment of cerebellar inhibitory synapses by an autoantibody to glutamate decarboxylase. J Neurol Sci. (2000) 175:404. doi: 10.1016/s0022-510x(00)00272-0

11. Budhram A, Leung A, Nicolle MW, Burneo JG. Diagnosing autoimmune limbic encephalitis. CMAJ. (2019) 191:E529-E34. doi: 10.1503/cmaj.181548

12. Albert DV, Pluto CP, Weber A, Vidaurre J, Barbar-Smiley F, Abdul Aziz R, et al. Utility of neurodiagnostic studies in the diagnosis of autoimmune encephalitis in children. Pediatr Neurol. (2016) 55:3745. doi: 10.1016/j.pediatrneurol.2015.10.016

13. Lancaster E, Lai M, Peng X, Hughes E, Constantinescu R, Raizer J, et al. Antibodies to the GABA(B) receptor in limbic encephalitis with seizures: case series and characterisation of the antigen. The Lancet Neurology. (2010) 9:67-76. doi: 10.1016/s1474-4422(09)70324-2

14. Grain R, Lally J, Stubbs B, Malik S, LeMince A, Nicholson TR, et al. Autoantibodies against voltage-gated potassium channel and glutamic acid decarboxylase in psychosis: a systematic review, meta-analysis, and case series. Psychiatry Clin Neurosci. (2017) 71:678-89. doi: 10.1111/pcn. 12543

15. Dalmau J, Armangue T, Planaguma J, Radosevic M, Mannara F, Leypoldt F, et al. An update on anti-NMDA receptor encephalitis for neurologists and psychiatrists: mechanisms and models. Lancet Neurol. (2019) 18:104557. doi: 10.1016/S1474-4422(19)30244-3

16. Boesen MS, Born AP, Lydolph MC, Blaabjerg M, Børresen ML. Pediatric autoimmune encephalitis in Denmark during 2011-17: a nationwide multicenter population-based cohort study. Eur J Paediatr Neurol. (2019) 23:639-52. doi: 10.1016/j.ejpn.2019.03.007

17. Herken J, Prüss H. Red flags: clinical signs for identifying autoimmune encephalitis in psychiatric patients. Front Psychiatry. (2017) 8:25. doi: $10.3389 /$ fpsyt.2017.00025

18. Pollak TA, Lennox BR, Müller S, Benros ME, Prüss H, van Elst LT, et al. Autoimmune psychosis: an international consensus on an approach to the diagnosis and management of psychosis of suspected autoimmune origin. Lancet Psych. (2020) 7:93-108. doi: 10.1016/S2215-0366(19) 30290-1
19. Najjar S, Pearlman D, Devinsky O, Najjar A, Nadkarni S, Butler T, et al. Neuropsychiatric autoimmune encephalitis without VGKC-complex, NMDAR, and GAD autoantibodies: case report and literature review. Cogn Behav Neurol. (2013) 26:36-49. doi: 10.1097/WNN.0b013e31828b6531

20. Mäkelä K-M, Hietaharju A, Brander A, Peltola J. Clinical management of epilepsy with glutamic acid decarboxylase antibody positivity: the interplay between immunotherapy and anti-epileptic drugs. Front Neurol. (2018) 9:579. doi: 10.3389/fneur.2018.00579

21. Li X, Guo Q, Zheng Z, Wang X, Liu S. Immune-mediated epilepsy with GAD65 antibodies. J Neuroimmunol. (2020) 341:577189. doi: 10.1016/j.jneuroim.2020.577189

22. Bien CG, Vincent A, Barnett MH, Becker AJ, Blümcke I, Graus F, et al. Immunopathology of autoantibody-associated encephalitides: clues for pathogenesis. Brain. (2012) 135:1622-38. doi: 10.1093/brain/aws082

23. Onugoren MD, Golombeck KS, Bien C, Abu-Tair M, Brand M, Bulla-Hellwig $M$, et al. Immunoadsorption therapy in autoimmune encephalitides. Neurol-Neuroimmunol Neuroinfl. (2016) 3:e207. doi: 10.1212/NXI.0000000000000207

24. Petit-Pedrol M, Armangue T, Peng X, Bataller L, Cellucci T, Davis R, et al. Encephalitis with refractory seizures, status epilepticus, and antibodies to the GABAA receptor: a case series, characterisation of the antigen, and analysis of the effects of antibodies. Lancet Neurol. (2014) 13:27686. doi: 10.1016/s1474-4422(13)70299-0

25. Stanley JA, Raz N. Functional magnetic resonance spectroscopy: the "New" MRS for cognitive neuroscience and psychiatry research. Front Psychiatry. (2018) 9:76. doi: 10.3389/fpsyt.2018.00076

26. Vogrig A, Joubert B, Ducray F, Thomas L, Izquierdo C, Decaestecker K, et al. Glioblastoma as differential diagnosis of autoimmune encephalitis. J Neurol. (2018) 265:669-77. doi: 10.1007/s00415-018-8767-1

27. Wagner J, Witt JA, Helmstaedter C, Malter MP, Weber B, Elger CE. Automated volumetry of the mesiotemporal structures in antibodyassociated limbic encephalitis. J Neurol Neurosurg Psychiatry. (2015) 86:73542. doi: 10.1136/jnnp-2014-307875

28. Wagner J, Schoene-Bake JC, Witt JA, Helmstaedter C, Malter MP, Stoecker $\mathrm{W}$, et al. Distinct white matter integrity in glutamic acid decarboxylase and voltage-gated potassium channel-complex antibody-associated limbic encephalitis. Epilepsia. (2016) 57:475-83. doi: 10.1111/epi.13297

Conflict of Interest: The authors declare that the research was conducted in the absence of any commercial or financial relationships that could be construed as a potential conflict of interest.

Publisher's Note: All claims expressed in this article are solely those of the authors and do not necessarily represent those of their affiliated organizations, or those of the publisher, the editors and the reviewers. Any product that may be evaluated in this article, or claim that may be made by its manufacturer, is not guaranteed or endorsed by the publisher.

Copyright (c) 2022 Wang, Gong, Kong, Cai, Huang, Zheng, Lin, Li and Zhang. This is an open-access article distributed under the terms of the Creative Commons Attribution License (CC BY). The use, distribution or reproduction in other forums is permitted, provided the original author(s) and the copyright owner(s) are credited and that the original publication in this journal is cited, in accordance with accepted academic practice. No use, distribution or reproduction is permitted which does not comply with these terms. 